\title{
COBORDISM CLASSES OF MANIFOLDS WITH CATEGORY FOUR
}

\author{
HARPREET SINGH
}

\begin{abstract}
The Lusternik-Schnirelmann category of a manifold $M$ is the smallest integer $k$ such that $M$ can be covered by $k$ open sets each of which is contractible in $M$. The classification up to cobordism of manifolds with category 3 was completed by the author in 1985 . The object of this paper is to attempt a similar classification of manifolds with category 4 .
\end{abstract}

Introduction. Let $M$ be an $n$-dimensional manifold. ${ }^{1}$ The LusternikSchnirelmann category of $M$, cat $(M)$, is the smallest integer $k$ such that $M$ can be covered by $k$ open sets each of which is contractible in $M$. In [3], Mielke showed that an $n$-dimensional manifold $M$ with $\operatorname{cat}(M) \leq 3$, and with $n \equiv 3(\bmod 4)$, is a boundary. The author, in [6], showed that one can determine the possible cobordism class of any manifold $M$ with $\operatorname{cat}(M) \leq 3$. The object of this paper is to determine the possible cobordism classes of manifolds $M$ with cat $(M) \leq 4$.

As in [6] the strategy is to use the fact that cat $(M)$ is an upper bound for the length of nonzero products of the Stiefel-Whitney classes of $M$ to determine the nonzero Stiefel-Whitney numbers, and hence the cobordism class of $M$. However, $\operatorname{cat}(M) \leq 4$ is a much weaker restriction than $\operatorname{cat}(M) \leq 3$, and thus the classification problem is a lot harder.

We begin $\S 1$ by recalling, from [6], the definition, and basic properties, of Poincare algebras and the cobordism category of a manifold $M$, denoted by cobcat $(M)$. In $\S 2$ we begin the investigation by looking at even-dimensional manifolds, and show that an even-dimensional, nonbounding manifold $M$, with $\operatorname{cobcat}(M) \leq 4$ and $\operatorname{dim}(M) \neq 2^{s}+2$ for any $s \geq 2$, is cobordant to a square $N \times N$, where $N$ is also nonbounding, and $\operatorname{cobcat}(N) \leq 4$. This along with other results reduces the problem to one of looking at the odd dimensions. We look at the odd dimensions in $\S 3$, and show that for many such dimensions $n$, with more than three terms in their 2-adic expansions, there is no nonbounding $n$-dimensional manifold $M$ with cat $(M) \leq 4$. Computations in small dinmensions suggest conjectures in the other dimensions. However, there does not seem to be a general way of handling these dimensions. Finally, in $\S 4$, we give some applications to finding lower bounds for the category of spin manifolds, and also show that a spin manifold $M$, with $\operatorname{cat}(M) \leq 4$, is nonoriented cobordant to the square of an oriented manifold.

Received by the editors July 1,1987 .

1980 Mathematics Subject Classification (1985 Revision). Primary 57R75, 55M30.

Key words and phrases. Cobordism, Lusternik-Schnirelmann category.

This work was supported in part by funds from the Foundation of the University of North Carolina at Charlotte and from the State of North Carolina.

${ }^{1}$ All manifolds are to be closed, smooth and compact. 
Parts of $\S 1$ and $\S 2$ are from the author's dissertation at the University of Virginia. The author would like to thank Dr. R. E. Stong for his support and guidance during the writing of the dissertation and for useful discussions afterwards. The author would also like to thank Dr. T. Yoshida of Hiroshima University for taking interest in this problem. The author would like to acknowledge that Dr. Yoshida independently proved a version of Proposition 2.8 conjectured to him by the author.

1. Preliminaries. We begin by recalling the concepts of cobordism category, introduced in [6], and Poincaré algebras [9].

Let $B O$ denote the universal space of the infinite orthogonal group. It is well known (see [5]) that the cohomology ${ }^{2}$ of $B O$ is the polynomial ring $\mathbf{Z}_{2}\left[W_{1}, W_{2}, \ldots\right]$, where $W_{i}$ is the $i$ th Stiefel-Whitney class. The Steenrod Squares act on $H^{*}(B O)$ with action given by: $\operatorname{Sq}^{i}\left(W_{j}\right)=0$ if $i>j$ and otherwise

$$
\mathrm{Sq}^{i}\left(W_{j}\right)=\sum_{k=0}^{i}\left(\begin{array}{c}
j-i-1+k \\
k
\end{array}\right) W_{i-k} W_{j+k} .
$$

The Wu classes, $v_{i}$, can be defined inductively by the equations $v_{0}=1$ and

$$
W_{i}=v_{i}+\mathrm{Sq}^{1}\left(v_{i-1}\right)+\cdots+\mathrm{Sq}^{i-1}\left(v_{1}\right)
$$

Let $M$ be an $n$-dimensional manifold. Then there exists (see [9]) a graded algebra $\mathfrak{p}^{*}$ and an epimorphism $f: H^{*}(B O) \rightarrow \mathfrak{p}^{*}$ satisfying:

(1) $x[M]=0$ iff $x=0$ in $\mathfrak{p}^{*}$ for all $x \in H^{n}(B O)$.

(2) $x=0$ in $\mathfrak{p}^{*}$ iff $x \cdot\left(\mathfrak{p}^{n-\operatorname{dim}(x)}\right)=0$. In view of $(1)$, we can restate this as: $x=0$ in $\mathfrak{p}^{*}$ iff all Stiefel-Whitney numbers divisible by $x$ are zero.

(3) If $M$ is nonbounding, then $\mathfrak{p}^{*}$ is an $n$-dimensional algebra with Poincaré duality. Otherwise, $\mathfrak{p}^{*}=0$.

(4) The Steenrod squares act on $\mathfrak{p}^{*}$ in the obvious way, and both the Cartan product formula and the $\mathrm{Wu}$ formula still hold in $\mathfrak{p}^{*}$ as does the relation between the Stiefel-Whitney classes and the Wu classes.

(5) $v_{i} x=\mathrm{Sq}^{i}(x)$ in $\mathfrak{p}^{*}$ if $i+\operatorname{dim}(x)=n$.

(6) $v_{j}=0$ in $\mathfrak{p}^{*}$ if $j>n / 2$.

(7) $v_{j}$ is decomposable in $\mathfrak{p}^{*}$ if $j \neq$ power of 2 .

Note. (1) and (2) essentially define $\mathfrak{p}^{*}$. (3) is a consequence of (1) and (2). (4) and (7) follow from the corresponding properties being true in $H^{*}(B O)$. (5) and (6) are the result of combining (1), (2), and the corresponding results in $H^{*}(M)$. $\mathfrak{p}^{*}$ will be called the Poincaré algebra associated with $M$.

DEFINITION. Let $\mathfrak{p}_{k}^{*}$ denote the ideal of $k$-decomposable elements in $\mathfrak{p}^{*}$. Then the cobordism category of $M$, denoted by $\operatorname{cobcat}(M)$, is the least integer $k$ such that $\mathfrak{p}_{k}^{*}=0$. As a trivial consequence of (2) we have the following results:

1.1. If cobcat $(M) \leq k$, then all $k$-decomposable and all $(k-1)$-decomposable elements not in the top dimension are zero in $\mathfrak{p}^{*}$.

1.2. $\operatorname{cobcat}(M) \leq k$ iff $W_{i_{1}} \cdot W_{i_{p}}[M]=0$ for all partitions $i_{1}+\cdots+i_{p}=n$ with $p \geq k$.

From this it follows that cobcat is a cobordism invariant, and cobcat $\left(M_{1} \cup M_{2}\right) \leq$ $\max \left(\operatorname{cobcat}\left(M_{1}\right), \operatorname{cobcat}\left(M_{2}\right)\right)$. Since $\operatorname{cat}(M)$ is an upper bound for the length of nonzero products in cohomology (see [2]), $\operatorname{cobcat}(M) \leq \operatorname{cat}(M)$.

We close this section by stating two results from [6] which will be needed later.

${ }^{2}$ Cohomology will always be $\bmod 2$. 
PROPOSITION 1.3. Let $M$ be an $n$-dimensional manifold, and let $c \in H^{1}(M)$. Let $N$ be the submanifold of $M$ dual to $c$, and let $c^{2} x[M]=0$ for all $x$ in the subalgebra of $H^{*}(M)$ generated by $c, W_{1}, \ldots, W_{n}$, where $\operatorname{dim}(x)=n-2$. Then $W_{i_{1}} \cdots W_{i_{p}}[N]=c W_{i_{1}} \cdots W_{i_{p}}[M]$ for any partition $i_{1}+\cdots+i_{p}=n-1$.

THEOREM 1.4. If $M$ is an n-dimensional manifold with cobcat $(M) \leq 3$, then either $M$ is a boundary or else one of the following is true:

(1) $n=2^{s}$ where $s \geq 1, W_{2^{s-1}}^{2}[M] \neq 0$, and $M$ is cobordant to $\left(\mathbf{R} P^{2}\right)^{2^{s-1}}$.

(2) $n=2^{r}\left(2^{s}+1\right)$ where $r \geq 0$ and $s \geq 2$, and $M$ is cobordant to $(\tilde{M})^{2^{r}}$ where $W_{2} W_{2^{s}-1}[\tilde{M}] \neq 0$, and $\tilde{M}=\bigcup_{j=0}^{2^{s-2}} P\left(1,2^{s-1}-2 j\right) \times N_{j}$ where $P(1, m)$ is the Dold manifold $\left(S^{1} \times \mathbf{C} P^{m}\right) / \mathbf{Z}_{2}, N_{j}$ is the degree $4 j$ term in the formal inverse $\left(1+\mathbf{C} P^{2}+\mathbf{C} P^{4}+\mathbf{C} P^{6}+\cdots\right)^{-1}$, and $\bigcup$ denotes disjoint union.

2. Even-dimensional manifolds with $\operatorname{cobcat}(M) \leq 4$. In the next two sections we will attempt to describe the cobordism class of an $n$-dimensional manifold $M$ with cobcat $(M) \leq 4$. Throughout the rest of the paper $\mathfrak{p}^{*}$ will denote the Poincaré algebra associated with such a manifold $M$, and $p_{2}^{*}$ will denote the ideal of decomposable elements. The symbol ( ) will denote a mod 2 binomial coefficient. Its entries will not be entered if they are irrelevant.

In this section we will show that we can reduce the problem to one of looking at odd-dimensional manifolds. In view of (1.2), we note that all we need to do is to examine the products $W_{i} W_{j} W_{k}$, where $i, j, k \geq 0$, and $i+j+k=n$. As a trivial consequence of the $\mathrm{Wu}$ formula we have:

2.1. $W_{2^{s}+k} \equiv \mathrm{Sq}^{k}\left(W_{2^{s}}\right) \operatorname{modp} \mathbf{p}_{2}^{*}$ where $2^{s}+k \leq n$, and $0 \leq k<2^{s}$.

We also have

2.2. $W_{2} \equiv v_{2} s \bmod \mathfrak{p}_{2}^{*}$.

LEMMA 2.3. $W_{1}^{2}=0$ in $\mathfrak{p}^{*}$ if $n \geq 3$.

Proof. $\mathrm{Sq}^{1}\left(W_{1}^{2}\right)=0$, and $\mathrm{Sq}^{2}\left(W_{1}^{2}\right)=W_{1}^{4}=0$ in $\mathfrak{p}^{*}$. Thus clearly $\mathrm{Sq}^{j}\left(W_{1}^{2}\right)=0$ if $j>0$. Let $n-2=2^{s}+k$, where $0 \leq k<2^{s}$.

If $k>0$, then

$$
\begin{aligned}
W_{1}^{2} W_{n-2} & =W_{1}^{2} \mathrm{Sq}^{k}\left(W_{2^{s}}\right)=\mathrm{Sq}^{k}\left(W_{1}^{2} W_{2^{s}}\right) \\
& =v_{k} W_{1}^{2} W_{2^{s}}=0 \quad \text { in } \mathfrak{p}^{*}
\end{aligned}
$$

If $k=0$, then

$$
\begin{aligned}
W_{1}^{2} W_{n-2} & =W_{1}^{2} W_{2^{s}}=W_{1}^{2} v_{2^{s}} \\
& =\mathrm{Sq}^{2^{s}}\left(W_{1}^{2}\right)=0 \text { in } \mathfrak{p}^{*} .
\end{aligned}
$$

LEMMA 2.4. $W_{1}=0$ in $\mathfrak{p}^{*}$ unless $n=2^{r}\left(2^{s}+1\right)+1$, where $r \geq 0$ and $s \geq 2$.

Proof. Let $N$ be the $(n-1)$-dimensional submanifold dual to $W_{1}(M)$. Then, by Proposition 1.3,

$$
W_{i_{1}} \cdots W_{i_{p}}[N]=W_{1} W_{i_{1}} \cdots W_{i_{p}}[M]
$$

for any partition $i_{1}+\cdots+i_{p}=n-1$. Thus cobcat $(N) \leq 3$, and $W_{1}(M)=0$ in $\mathfrak{p}^{*}$ iff $N$ is a boundary. By Theorem $1.4, N$ is a boundary unless $n-1$ is $2^{t}$ or $2^{r}\left(2^{s}+1\right)$, where $t \geq 1, r \geq 0$, and $s \geq 2$. Furthermore if $n-1=2^{t}$, and $N$ is not a boundary, then $N$ is cobordant to $\left(\mathbf{R} P^{2}\right)^{2^{t-1}}$, and $W_{2^{t-1}}^{2}[N] \neq 0$. However, 
$W_{2^{t-1}}^{2}[N]=W_{1} W_{2^{t-1}}^{2}[M]$, and $W_{1} W_{2^{t-1}}^{2}=\operatorname{Sq}^{1}\left(W_{2^{t-1}}^{2}\right)=0$. Thus $N$ is a boundary even in the case $n-1=2^{t}$.

LEMMA 2.5. If $W_{1}=0$ in $\mathfrak{p}^{*}$, then:

(1) $W_{2 j+1}=\mathrm{Sq}^{1}\left(W_{2 j}\right)$ in $\mathfrak{p}^{*}$.

(2) $\mathrm{Sq}^{1}(x) \mathrm{Sq}^{1}(y)=0$ in $\mathfrak{p}^{*}$ if $\operatorname{dim}(x)+\operatorname{dim}(y)+2=n$.

ProOF. (1) $\mathrm{Sq}^{1}\left(W_{2 j}\right)=W_{1} W_{2 j}+W_{2 j+1}$.

(2) $\operatorname{Sq}^{1}(x) \operatorname{Sq}^{1}(y)=\operatorname{Sq}^{1}\left(x \operatorname{Sq}^{1}(y)\right)=W_{1} x \operatorname{Sq}^{1}(y)=0$ in $p^{*}$.

LEMMA 2.6. If $n$ is even, $n>2$, and $W_{1}=0$ in $\mathfrak{p}^{*}$, then:

(1) $W_{i} W_{n-i}=0$ in $\mathfrak{p}^{*}$ for all odd $i$.

(2) $W_{2} \operatorname{Sq}^{1}(x)=0$ in $\mathfrak{p}^{*}$ if $\operatorname{dim}(x)=n-3$.

ProOF. (1) Since $W_{1}=0$, we may assume that $1<i<n-1$. Then $W_{i} W_{n-i}=$ $\mathrm{Sq}^{1}\left(W_{i-1}\right) \mathrm{Sq}^{1}\left(W_{n-i-1}\right)=0$ in $\mathfrak{p}^{*}$ by Lemma 2.5 .

(2) $\mathrm{Sq}^{1}\left(W_{n-3}\right)=W_{1} W_{n-3}$ since $n-3$ is odd. Thus $W_{2} \operatorname{Sq}^{1}\left(W_{n-3}\right)=W_{2} W_{1} W_{n-3}$ $=0$ in $\mathfrak{p}^{*}$. Let $n-3=j+k$, and assume that $j$ is odd. Then

$$
W_{2} \operatorname{Sq}^{1}\left(W_{j} W_{k}\right)=W_{2} W_{j} W_{k+1}=\operatorname{Sq}^{2}\left(W_{j}\right) W_{k+1}+W_{j} \operatorname{Sq}^{2}\left(W_{k+1}\right) .
$$

By the Wu formula

$$
\mathrm{Sq}^{2}\left(W_{i}\right)=W_{2} W_{i}+\left(\begin{array}{c}
i-1 \\
2
\end{array}\right) W_{i+2} \text { in } \mathfrak{p}^{*} \text { for all } i \geq 2 .
$$

Thus

$$
W_{2} \operatorname{Sq}^{1}\left(W_{j} W_{k}\right)=\left(\begin{array}{c}
j-1 \\
2
\end{array}\right) W_{j+2} W_{k+1}+\left(\begin{array}{l}
k \\
2
\end{array}\right) W_{k+3} W_{j}=0
$$

in $\mathfrak{p}^{*}$. Also, $W_{2} \operatorname{Sq}^{1}(x)=0$ in $\mathfrak{p}^{*}$ if $\operatorname{dim}(x)=n-3$, and $x$ is 3-decomposable.

LEMMA 2.7. Let $r+j+k<n$. Then

$$
\mathrm{Sq}^{r}\left(W_{j} W_{k}\right)=\sum_{\beta=0}^{r}\left(\begin{array}{c}
j-1 \\
\beta
\end{array}\right)\left(\begin{array}{c}
k-1 \\
r-\beta
\end{array}\right) W_{j+\beta} W_{k+r-\beta} \text { in } \mathfrak{p}^{*}
$$

PROOF.

$$
\mathrm{Sq}^{r}\left(W_{j} W_{k}\right)=\sum_{\beta=0}^{r} \mathrm{Sq}^{\beta}\left(W_{j}\right) \mathrm{Sq}^{r-\beta}\left(W_{k}\right)
$$

by the Cartan formula. Also, $\mathrm{Sq}^{\beta}\left(W_{j}\right) \equiv\left(\begin{array}{c}j-1 \\ \beta\end{array}\right) W_{j+\beta} \bmod \mathrm{p}_{2}^{*}$ for any $\beta, j$. Thus the result follows since 4-decomposable elements and 3-decomposable elements not in top dimension are zero.

PROPOSITION 2.8. If $n$ is even, $n>2$, and $W_{1}=0$ in $\mathfrak{p}^{*}$, then $W_{j}=0$ in $\mathfrak{p}^{*}$ for all odd $j$.

ProOF. Since $W_{1}=0$, we may assume $j>1$. By Lemma $2.6, W_{j} W_{n-j}=0$ and clearly $W_{j} x=0$ in $\mathfrak{p}^{*}$ if $x$ is 3-decomposable. Thus all we need to do is to show that $W_{j} x=0$ in $\mathfrak{p}^{*}$ if $x \in \mathfrak{p}_{2}^{*}$ and $\operatorname{dim}(x)=n-j$.

(1) Let $k=n-j-2$. Thus $k$ is odd, and so $W_{j} W_{2} W_{k}=W_{2} \operatorname{Sq}^{1}\left(W_{j-1} W_{k}\right)=0$ in $\mathfrak{p}^{*}$ by Lemma 2.6 . 
(2) Let $k=n-j-2^{a}$ where $a \geq 2$ and $k>0$. Thus $k$ is odd, and so

$$
W_{j} W_{2^{a}} W_{k}=W_{2^{a}} \mathrm{Sq}^{1}\left(W_{j-1} W_{k}\right)=\mathrm{Sq}^{2^{a}} \mathrm{Sq}^{1}\left(W_{j-1} W_{k}\right) .
$$

By the Adem relations,

$$
\mathrm{Sq}^{2} \mathrm{Sq}^{2^{a}-1}=\mathrm{Sq}^{2^{a}+1}+\mathrm{Sq}^{2^{a}} \mathrm{Sq}^{1}
$$

and so

$$
\begin{aligned}
W_{j} W_{2^{a}} W_{k} & =\mathrm{Sq}^{2} \mathrm{Sq}^{2^{a}-1}\left(W_{j-1} W_{k}\right)+\mathrm{Sq}^{1} \mathrm{Sq}^{2^{a}}\left(W_{j-1} W_{k}\right) \\
& =W_{2} \mathrm{Sq}^{1} \mathrm{Sq}^{2^{a}-2}\left(W_{j-1} W_{k}\right)+W_{1} \mathrm{Sq}^{2^{a}}\left(W_{j-1} W_{k}\right) \\
& =0 \quad \text { in } \mathfrak{p}^{*} \text { by Lemma } 2.6 .
\end{aligned}
$$

Thus from (1) and (2) $W_{j^{\prime}} W_{2^{a}}=0$ in $\mathfrak{p}^{*}$ for any odd $j^{\prime}$ and any $a \geq 0$. Assume inductively that $W_{j^{\prime}} W_{2^{a}+t}=0$ for any odd $j^{\prime}$, and any $t<b$. Let $k=n-j-2^{a}-b$, where $a \geq 1,0<b<2^{a}$, and $k>0$. Then by the Cartan formula and Lemma 2.7,

$$
\begin{aligned}
W_{j} W_{2^{a}+b} W_{k} & =\operatorname{Sq}^{b}\left(W_{2^{a}}\right) W_{j} W_{k}=\sum_{\alpha=0}^{b-1} \mathrm{Sq}^{\alpha}\left(W_{2^{a}}\right) \mathrm{Sq}^{b-\alpha}\left(W_{j} W_{k}\right) \\
& =\sum_{\alpha=0}^{b-1} \sum_{\beta=0}^{b-\alpha}\left(\begin{array}{c}
j-1 \\
\beta
\end{array}\right)\left(\begin{array}{c}
k-1 \\
b-\alpha-\beta
\end{array}\right) W_{2^{a}+\alpha} W_{j+\beta} W_{k+b-\alpha-\beta} .
\end{aligned}
$$

If $\beta$ is odd, then $\left(\begin{array}{c}j-1 \\ \beta\end{array}\right)=0$ and if $\beta$ is even, then $j+\beta$ is odd and so by the induction hypothesis

$$
W_{2^{a}+\alpha} W_{j+\beta} W_{k+b-\alpha-\beta}=0 \text { in } \mathfrak{p}^{*} .
$$

Thus $W_{j} W_{2^{a}+b} W_{k}=0$ in $\mathfrak{p}^{*}$.

PROPOSITION 2.9. Let $M$ be a nonbounding n-dimensional manifold with $\operatorname{cobcat}(M) \leq 4$. If $n$ is even, $n>2$, and $W_{1}=0$ in $\mathfrak{p}^{*}$, then $M$ is cobordant to $N \times N$ where $N$ is also nonbounding and $\operatorname{cobcat}(N) \leq 4$.

Proof. $W_{1}=0$ in $\mathfrak{p}^{*}$, and so by Proposition $2.8, W_{j}=0$ in $\mathfrak{p}^{*}$ for all odd $j$. Thus $M$ is cobordant to a square $N \times N$ (see $[4,6]$ ), with $W_{i_{1}} \cdots W_{i_{p}}[N]=$ $W_{2 i_{1}} \cdots W_{2 i_{p}}[M]$ for any partition $i_{1}+\cdots+i_{p}=n / 2$. Thus clearly $N$ is nonbounding, and cobcat $(N) \leq 4$.

COROLLARY 2.10. Let $M$ be a nonbounding n-dimensional manifold with $\operatorname{cobcat}(M) \leq 4$, and let $n=2^{t} m$ where $t \geq 1$ and $m>1$.

(1) If $m \neq 2^{s}+1$ for any $s \geq 1$, then $M$ is cobordant to $(N)^{2^{t}}$ where $N$ is a nonbounding, $m$-dimensional manifold with $\operatorname{cobcat}(N) \leq 4$.

(2) If $m=2^{s}+1$ for some $s \geq 1$, then $M$ is cobordant to $(N)^{2^{t-1}}$ where $N$ is a nonbounding, 2m-dimensional manifold with $\operatorname{cobcat}(N) \leq 4$.

ProOF. By Lemma 2.4, $W_{1}(M)=0$ in $\mathfrak{p}^{*}$ if $\operatorname{dim}(M)=2 r$, where $r \neq 2^{s}+1$ for any $s \geq 1$. The result follows by inductively applying Proposition 2.9.

COROLLARY 2.11. Let $M$ be a $2^{t}$-dimensional manifold with cobcat $(M) \leq 4$, and $t \geq 1$. Then $M$ is either a boundary, or $M$ is cobordant to $\left(\mathbf{R} P^{2}\right)^{2^{t-1}}$.

PROOF. If $M$ is not a boundary, then by Corollary $2.10, M$ is cobordant to $(N)^{2^{t-1}}$ where $N$ is a nonbounding 2-dimensional manifold with cobcat $(N) \leq 4$. Then $N$ is clearly cobordant to $\mathbf{R} P^{2}$. 
PROPOSITION 2.12. If $n=2^{s}+2$ where $s \geq 2$, and if $W_{1} W_{2} W_{2^{s}-1}[M]=$ 0 , then $M$ is either a boundary or else $M$ is cobordant to $N \times N$ where $N$ is a nonbounding $\left(2^{s-1}+1\right)$-dimensional manifold with cobcat $(N) \leq 4$.

Proof. Suppose $M$ is not a boundary, and let $M^{\prime}$ be the $\left(2^{s}+1\right)$ - dimensional submanifold of $M$ dual to $W_{1}(M)$. Then as in Lemma 2.4, $\operatorname{cobcat}\left(M^{\prime}\right) \leq 3$, and $W_{2} W_{2^{s}-1}\left[M^{\prime}\right]=W_{1} W_{2} W_{2^{s}-1}[M]=0$. Thus by Theorem $1.4, M^{\prime}$ is a boundary, and so $W_{1} W_{j} W_{n-1-j}[M]=W_{j} W_{n-1-j}\left[M^{\prime}\right]=0$ in $\mathfrak{p}^{*}$ for all $0 \leq j \leq n-1$, and so by Proposition 2.9, we have the result.

COROLLARY 2.13. Let $M_{1}$ and $M_{2}$ be two nonbounding $\left(2^{s}+2\right)$-dimensional manifolds, where $s \geq 2$, cobcat $\left(M_{1}\right) \leq 4$, and cobcat $\left(M_{2}\right) \leq 4$. If neither $M_{1}$ nor $M_{2}$ is a square, then $M_{1}=M_{2} \cup(N \times N)$ where $\operatorname{cobcat}(N) \leq 4$.

PrOOF. By Proposition 2.12,

$$
W_{1} W_{2} W_{2^{s}-1}\left[M_{1}\right]=W_{1} W_{2} W_{2^{s}-1}\left[M_{2}\right]=1,
$$

and so $W_{1} W_{2} W_{2^{s}-1}\left[M_{1} \cup M_{2}\right]=0$. It is easily seen that cobcat $\left[M_{1} \cup M_{2}\right] \leq 4$. The result follows from Proposition 2.12.

There exist classes $s_{k} \in H^{k}(B O)$ (see [10]) defined inductively by Newton's formula: $s_{k}+W_{1} s_{k-1}+\cdots+W_{k-1} s_{1}+[k]_{2} W_{k}=0$ for $k>0$, where $[k]_{2}$ is the $\bmod$ 2 reduction of $k$, and $s_{0}=1$. These classes have the property that a cobordism class represented by an $n$-dimensional manifold $M$ is decomposable iff $s_{n}[M]=0$. It can be shown that $\mathrm{Sq}^{j}\left(s_{k}\right)=\left(\begin{array}{c}k \\ j\end{array}\right) s_{k+j}$ for all $k \geq j>0$.

COROLLARY 2.14. Let $n=2^{r}+2$, where $r \geq 2$, and let $M$ be a nonbounding, $n$-dimensional manifold which is not cobordant to a square. Then $M$ is indecomposable.

PROOF. $s_{2^{r}+2}=\mathrm{Sq}^{3}\left(s_{2^{r}-1}\right)=v_{3} s_{2^{r}-1}$. Moreover $v_{3}$ is decomposable, and $s_{2^{r}-1} \equiv W_{2^{r}-1} \bmod p_{2}^{*}$. It follows that $s_{2^{r}+2}=v_{3} W_{2^{r}-1}=\left(W_{3}+\operatorname{Sq}^{1}\left(W_{2}\right)\right) W_{2^{r}-1}=$ $W_{1} W_{2} W_{2^{r}-1}$ in $\mathfrak{p}^{*}$. By hypothesis, and Proposition $2.12, W_{1} W_{2} W_{2^{r-1}} \neq 0$ in $\mathfrak{p}^{*}$, and so $M$ is indecomposable.

3. Odd dimensional manifolds with $\operatorname{cobcat}(M) \leq 4$. The results of $\S 2$ have reduced the problem to one of looking at the odd-dimensional manifolds. The most tractable of these dimensions seem to be those $n$ for which $\alpha(n) \geq 4$, where $\alpha(n)$ is the number of nonzero terms in the 2-adic expansion of $n$.

LEMMA 3.1. If $n$ is odd, then:

(1) $x^{2}=0$ in $\mathfrak{p}^{*}$ for any $x$, where $\operatorname{dim}(x)>0$.

(2) $W_{\text {odd }} W_{\text {odd }}=0$ in $\mathfrak{p}^{*}$.

ProOF. (1) Let $0<2 i<n$. $n-2 i$ is odd, and so $W_{n-2 i}=\operatorname{Sq}^{1}\left(W_{n-2 i-1}\right)+$ $W_{1} W_{n-2 i-1}$. Hence,

$$
\begin{aligned}
W_{i}^{2} W_{n-2 i} & =W_{i}^{2} \operatorname{Sq}^{1}\left(W_{n-2 i-1}\right)+W_{i}^{2} W_{1} W_{n-2 i-1} \\
& =\operatorname{Sq}^{1}\left(W_{i}^{2} W_{n-2 i-1}\right)+\operatorname{Sq}^{1}\left(W_{i}^{2} W_{n-2 i-1}\right)=0
\end{aligned}
$$

in $\mathfrak{p}^{*}$, and so, $W_{i}^{2}=0$ in $\mathfrak{p}^{*}$ for all $i \geq 1$. It follows that $x^{2}=0$ in $\mathfrak{p}^{*}$ for all $x$ in $\mathfrak{p}^{*}$. 
(2) Let $i, j$ be odd and let $0<i, j<n$. Then

$$
\begin{aligned}
W_{i} W_{j} W_{n-i-j} & =W_{i} W_{j} \operatorname{Sq}^{1}\left(W_{n-i-j-1}\right)+W_{i} W_{j} W_{1} W_{n-i-j-1} \\
& =\mathrm{Sq}^{1}\left(W_{i} W_{j} W_{n-i-j-1}\right)+\operatorname{Sq}^{1}\left(W_{i} W_{j} W_{n-i-j-1}\right) \\
& =0
\end{aligned}
$$

in $\mathfrak{p}^{*}$, and so $W_{i} W_{j}=0$ in $\mathfrak{p}^{*}$.

PROPOSITION 3.2. Let $n=2^{r}+m$, where $0<m<2^{r}$, and let $\alpha(n) \geq 4$. Then $W_{2^{r}+k}=0$ in $\mathfrak{p}^{*}$, for any $0 \leq k \leq m$.

PROOF. $W_{2^{r}+k} \equiv \mathrm{Sq}^{k}\left(W_{2^{r}}\right) \equiv \mathrm{Sq}^{k}\left(v_{2^{r}}\right) \bmod \mathfrak{p}_{2}^{*}$. Moreover, $v_{2^{r}}=0$, and so, $W_{2^{r}+k}$ is decomposable for each $0 \leq k \leq m$. Hence, $W_{2^{r}+k} x=0$ in $\mathfrak{p}^{*}$ if $x$ is decomposable.

It is easy to see that $W_{n}=0$, and $W_{1} W_{n-1}=0$. Moreover,

$$
\begin{aligned}
W_{2} W_{n-2} & =\mathrm{Sq}^{2} \mathrm{Sq}^{(n-3) / 2}\left(v_{(n-1) / 2}\right) \\
& =\mathrm{Sq}^{(n-1) / 2} \mathrm{Sq}^{1}\left(v_{(n-1) / 2}\right) .
\end{aligned}
$$

Since $\alpha(n) \geq 4,(n-1) / 2$ is not a power of 2 , and so $v_{(n-1) / 2}$ is decomposable. Thus, $W_{2} W_{n-2}=0$.

Assume inductively that $W_{i} W_{n-i}=0$ for all $0 \leq i<j$, where $2<j \leq m$. Then:

$$
W_{j} W_{n-j}=\mathrm{Sq}^{j}\left(W_{n-j}\right)+\sum_{t=1}^{j}() W_{j-t} W_{n-j+t},
$$

and so by the induction hypothesis, $W_{j} W_{n-j}=\mathrm{Sq}^{j}\left(W_{n-j}\right)=v_{j} W_{n-j} . W_{n-j}=$ $W_{2^{r}+m-j}$ is decomposable, and so is $v_{j}$ if $j$ is not a power of 2 , and so in this case $W_{j} W_{n-j}=0$. If $j=2^{s}$, for some $s \geq 2$, then

$$
\begin{aligned}
W_{j} W_{n-j} & =\mathrm{Sq}^{2^{s}}\left(W_{n-2^{s}}\right)=\mathrm{Sq}^{2^{s}} \mathrm{Sq}^{1}\left(W_{n-2^{s}-1}\right) \\
& =\mathrm{Sq}^{1} \mathrm{Sq}^{2^{s}}\left(W_{n-2^{s}-1}\right)+\mathrm{Sq}^{2} \mathrm{Sq}^{2^{s}-1}\left(W_{n-2^{s}-1}\right) \\
& =W_{1} \mathrm{Sq}^{2^{s}}\left(W_{n-2^{s}-1}\right)+\sum_{t=0}^{2^{s}-1}\left({ }_{t}\right) W_{2} W_{2^{s-1-t}} W_{n-2^{s}-1+t} .
\end{aligned}
$$

Since $\alpha(n) \geq 4, W_{1}=0$ in $\mathfrak{p}^{*}$. Moreover, $2^{s} \leq m$ implies that $2^{s}+1 \leq m$, and so, $W_{n-2^{s}-1+t}$ is decomposable for all $0 \leq t \leq 2^{s}-1$. We also know that $W_{2} W_{n-2}=0$. Thus, $W_{j} W_{n-j}=0$ for all $0 \leq j \leq m$.

THEOREM 3.3. Let $n$ be odd, and let $W_{j} W_{n-j}=0$ for all $0 \leq j \leq n$. Then $M$ is a boundary.

PROOF. Let $i=\min (i, j, k)$, where $i, j, k \geq 0$ and $i+j+k=n$. By hypothesis, $W_{i} W_{j} W_{k}=0$ if $i=0$. Let $0<i<n$, and assume inductively that $W_{p} W_{q} W_{r}=0$ if $\min (p, q, r)<i$, where $p, q, r \geq 0$ and $p+q+r=n$.

By Lemma 3.1, we may assume that $i<\min (j, k)$. If $i=2^{a}$, then

$$
\begin{aligned}
W_{2^{a}} W_{j} W_{k} & =\mathrm{Sq}^{2^{a}}\left(W_{j} W_{k}\right)=\sum_{\alpha=0}^{2^{a}} \mathrm{Sq}^{\alpha}\left(W_{j}\right) \mathrm{Sq}^{2^{a}-\alpha}\left(W_{k}\right) \\
& =\sum_{\alpha=0}^{2^{a}} \sum_{t=0}^{\alpha}\left({ }_{t}\right) W_{\alpha-t} W_{j+t} \mathrm{Sq}^{2^{a}-\alpha}\left(W_{k}\right) .
\end{aligned}
$$


By the induction hypothesis, $W_{\alpha-t} W_{j+t} \mathrm{Sq}^{2^{a}-\alpha}\left(W_{k}\right)=0$ in $\mathfrak{p}^{*}$ if $0<\alpha-t<2^{a}$, and $\alpha-t=2^{a}$ iff $\alpha=2^{a}$, and $t=0$. Thus,

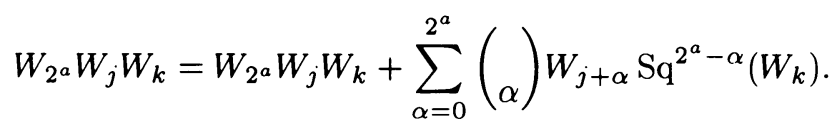

As above, we can expand $\mathrm{Sq}^{2}-\alpha\left(W_{k}\right)$, and conclude that

$$
W_{2^{a}} W_{j} W_{k}=W_{2^{a}} W_{j} W_{k}+W_{j} W_{2^{a}} W_{k}+\sum_{\alpha=0}^{2^{a}}() W_{j+\alpha} W_{k+2^{a}-\alpha}
$$

Thus by hypothesis, $W_{2^{a}} W_{j} W_{k}=0$.

If $i=2^{a}+b$, then by Lemma 2.7 ,

$$
\begin{aligned}
W_{2^{a}+b} W_{j} W_{k} & =\operatorname{Sq}^{b}\left(W_{2^{a}}\right) W_{j} W_{k}=\sum_{\alpha=0}^{b-1} \mathrm{Sq}^{\alpha}\left(W_{2^{a}}\right) \mathrm{Sq}^{b-\alpha}\left(W_{j} W_{k}\right) \\
& =\sum_{\alpha=0}^{b-1} \sum_{\beta=0}^{b-\alpha}\left(\begin{array}{c}
j-1 \\
\beta
\end{array}\right)\left(\begin{array}{c}
k-1 \\
b-\alpha-\beta
\end{array}\right) W_{2^{a}+\alpha} W_{j+\beta} W_{k+b-\alpha-\beta}=0
\end{aligned}
$$

by the induction hypothesis.

COROLLARY 3.4. If $n=2^{r+1}-1$, where $r \geq 3$, then $M$ is a boundary.

PROOF. For any $0 \leq k \leq n$, either $k \geq 2^{r}$, or $n-k \geq 2^{r}$. Thus, by Proposition $3.2, W_{k} W_{n-k}=0$ for all $0 \leq k \leq n$, and so by Theorem $3.3, M$ is a boundary.

LEMMA 3.5. If $n$ is odd, $W_{1}=0$ in $\mathfrak{p}^{*}$, and $M$ is decomposable, then the $W u$ class $v_{j}=0$ in $\mathfrak{p}^{*}$ whenever $j$ is not a power of two.

Proof. $v_{j}$ is decomposable, and so $v_{j} x=0$ for any $x \in \mathfrak{p}_{2}^{*}$. $\mathfrak{p}^{*}$.

If $j$ is odd, then $v_{j} W_{n-j}=\operatorname{Sq}^{j}\left(W_{n-j}\right)=\operatorname{Sq}^{1} \operatorname{Sq}^{j-1}\left(W_{n-j}\right)=0$ since $W_{1}=0$ in

If $j$ is even, then $n-j$ is odd, and so by the Newton formula, $W_{n-j} \equiv s_{n-j}$ $\bmod \mathfrak{p}_{2}^{*}$. Hence, $v_{j} W_{n-j}=v_{j} s_{n-j}=\operatorname{Sq}^{j}\left(s_{n-j}\right)=(\quad) s_{n}=0$ since $M$ is decomposable.

LEMMA 3.6. If $v_{k} W_{n-k}=0$ for all $0 \leq k \leq n$, then $W_{k} W_{n-k}=0$ for all $0 \leq k \leq n$.

PROOF. $W_{1} W_{n-1}=v_{1} W_{n-1}=0$. Let $1<k<n$, and assume inductively that $W_{i} W_{n-i}=0$ for all $0 \leq i<k$. Then $W_{k} W_{n-k}=\operatorname{Sq}^{k}\left(W_{n-k}\right)$ by the induction hypothesis, and so $W_{k} W_{n-k}=v_{k} W_{n-k}=0$.

Proposition 3.7. Let $n=2^{a}(p)+\left(2^{t}-1\right)$, where $p$ is odd, $1 \leq t<a$, and $\alpha(p) \geq 3$. Then $M$ is decomposable.

PROOF. We can write $n=2^{r}+2^{a}(m)+\left(2^{t}-1\right)$, where $2^{a}(m)<2^{r}, m$ is odd, and $\alpha(m) \geq 2$. Thus

$$
\mathrm{Sq}^{2^{a}+2^{t}}\left(s_{2^{r}+2^{a}(m-1)-1}\right)=\left(\begin{array}{c}
2^{r}+2^{a}(m-1)-1 \\
2^{a}+2^{t}
\end{array}\right) s_{n} .
$$


Moreover, since $m$ is odd and $m \geq 3$,

$$
2^{r}+2^{a}(m-1)-1=2^{r}+2^{a}(m-3)+\left(2^{a+1}-1\right),
$$

and so the binomial coefficient above is nonzero. Thus $s_{n}=v_{2^{a}+2^{t}} s_{2^{r}+2^{a}(m-1)-1}$. Now, $v_{2^{a}+2^{t}}$ is decomposable, and modulo decomposables

$$
s_{2^{r}+2^{a}(m-1)-1}=W_{2^{r}+2^{a}(m-1)-1}
$$

and this is zero in $\mathfrak{p}^{*}$ by Proposition 3.2. Thus $s_{n}=0$.

THEOREM 3.8. Let $n=2^{r}+2^{r-1}+m$, where $m$ is odd, $m<2^{r-1}, m+1$ is not a power of two, and $\alpha(m) \geq 2$. Then $M$ is a boundary.

PROOF. $v_{2^{r}}=0$. If $2^{a}<2^{r}$, then $n-2^{a}>2^{r}$, and so by Proposition 3.2, $v_{2^{a}} W_{n-2^{a}}=0$. Moreover, $M$ is decomposable by Proposition 3.7. It follows then from Lemma 3.5 and Lemma 3.6 that $W_{k} W_{n-k}=0$ for all $0 \leq k \leq n$, and so by Theorem 3.3, $M$ is a boundary.

There seems to be no general way of handling the other odd dimensions, but calculations in small dimensions suggest the following conjectures for an $n$-dimensional manifold $M$ with $\operatorname{cobcat}(M) \leq 4$ :

1. If $\alpha(n) \geq 4$, then $M$ is a boundary.

2. If $\alpha(n)=3$, then there is a manifold $M_{1}$ with cobcat $\left(M_{1}\right) \leq 4$, such that, $M$ is either a boundary, or $M$ is cobordant to $M_{1}$.

3. If $n=2^{s}+1$, then $\operatorname{cobcat}(M) \leq 3$, and so $M$ is the manifold in Theorem 1.4.

4. Applications. We finish by giving a few applications to finding lower bounds for the category of nonbounding spin manifolds.

LEMMA 4.1. Let $n$ be odd. Then $W_{1}$ and $W_{2}$ are zero in $\mathfrak{p}^{*}$ iff $W_{k} W_{n-k}=0$ for all $0 \leq k \leq n$.

Proof. Since $n$ is odd, $W_{n}=0$.

$(\Rightarrow)$ By hypothesis, $W_{1} W_{n-1}=W_{2} W_{n-2}=0$. Assume inductively that $W_{i} W_{n-i}$ $=0$ for all $0 \leq i<k$, where $2<k \leq n$. Then

$$
\begin{aligned}
W_{k} W_{n-k} & =\mathrm{Sq}^{k}\left(W_{n-k}\right)+\sum_{t=1}^{k}() W_{k-t} W_{n-k+t} \\
& =\mathrm{Sq}^{k}\left(W_{n-k}\right) \quad \text { by induction hypothesis. }
\end{aligned}
$$

If $k$ is odd, then $\mathrm{Sq}^{k}=\mathrm{Sq}^{1} \mathrm{Sq}^{k-1}$, and since $W_{1}=0$ in $\mathfrak{p}^{*}, W_{k} W_{n-k}=0$. If $k$ is even, then $W_{k} W_{n-k}=\mathrm{Sq}^{k} \mathrm{Sq}^{1}\left(W_{n-k-1}\right)$, and,

$$
\mathrm{Sq}^{k} \mathrm{Sq}^{1}=\mathrm{Sq}^{2} \mathrm{Sq}^{k-1}+\left(\begin{array}{c}
k-2 \\
2
\end{array}\right) \mathrm{Sq}^{1} \mathrm{Sq}^{k}
$$

Thus $W_{k} W_{n-k}=0$ since, $W_{2}=W_{1}=0$ in $\mathfrak{p}^{*}$.

$(\Leftarrow) W_{1} W_{n-1}=W_{2} W_{n-2}=0$ by hypothesis, and it is easy to see that

$$
W_{1} W_{j} W_{n-j-1}=0
$$

if $j$ is odd, and otherwise, $W_{1} W_{j} W_{n-j-1}=W_{j+1} W_{n-j-1}+W_{j} W_{n-j}=0$ by hypothesis. Likewise, $W_{2} W_{j} W_{n-j-2}=() W_{j+2} W_{n-j-2}+() W_{j} W_{n-j}=0$. 
This result gives an interesting lower bound on the category of odd-dimensional, nonbounding, spin manifolds. We recall (see [8]), that if the cobordism class of $M$ can be represented by a spin manifold, then all Stiefel-Whitney numbers divisible by $W_{1}$ and $W_{2}$ are zero. If $\operatorname{cat}(M) \leq 4$, then, by Lemma 4.1 and Theorem 3.3, $M$ is a boundary. Hence we have the following.

COROLLARY 4.2. Let $M$ be an odd-dimensional spin manifold, which does not bound in $\mathfrak{N}_{*}$. Then $\operatorname{cat}(M) \geq 5$.

By Milnor [4], we have to go up to dimension 29 before we can find such a manifold.

In [4], Milnor conjectured that the square of an orientable manifold is nonoriented cobordant to a spin manifold. This was subsequently proven by Anderson [1]. A natural question to ask is if the converse is true, i.e. is every spin manifold nonoriented cobordant to a square of an orientable manifold? It is shown in [4] that this is true in dimensions less than 24, but not in dimension 24. Proposition 2.9 provides us with the following converse for manifolds with cat $(M) \leq 4$.

COROLlARY 4.3. Let $M$ be an even-dimensional, spin manifold which does not bound in $\mathfrak{N}_{*}$, and let cat $(M) \leq 4$. Then $M$ is nonoriented cobordant to a square of an oriented manifold.

ProOF. By Proposition 2.9, $M$ is nonoriented cobordant to a square $N \times N$ and since $W_{2}(M)=0$ in $\mathfrak{p}^{*}, N$ is cobordant to an orientable manifold.

Of course if $M$ is not a square, then cat $(M) \geq 5$. Such a manifold does exist in dimension 24 (see [4]). As in Corollary 4.3, we can show that if $M$ is an orientable manifold which does not bound in $\mathfrak{N}_{*}$, and $\operatorname{cat}(M) \leq 4$, then $M$ is unoriented cobordant to a square.

\section{REFERENCES}

1. P. G. Anderson, Cobordism classes of squares of orientable manifolds, Bull. Amer. Math. Soc. 70 (1964), 818-819.

2. I. M. James, On category, in the sense of Lusternik-Schnirelmann, Topology 17 (1978), 331-348.

3. M. V. Mielke, Cobordism properties of manifolds of small category, Proc. Amer. Math. Soc. 21 (1969), 332-334.

4. J. W. Milnor, On the Stiefel-Whitney numbers of complex manifolds and of spin manifolds, Topology 3 (1965), 223-230.

5. J. W. Milnor and J. D. Stasheff, Characteristic classes, Ann. of Math. Studies, no. 76, Princeton Univ. Press, Princeton, N.J., 1974.

6. H. Singh, Lusternik-Schnirelmann category and cobordism, Proc. Amer. Math. Soc. 102 (1988), $183-190$.

7. N. E. Steenrod and D. B. A. Epstein, Cohomology operations, Ann. of Math. Studies, no. 50, Princeton Univ. Press, Princeton, N.J., 1962.

8. R. E. Stong, Notes on cobordism theory, Math. Notes, Princeton Univ. Press, Princeton, N.J., 1968.

9. __ C'obordism and Stiefel-Whitney numbers, Topology 4 (1965), 241-256.

10. R. Thom, Quelques propriétés globales des variétés différentiables, Comment. Math. Helv. 28 (1954), 1786.

11. C. T. C. Wall, Determination of the cobordism ring, Ann. of Math. 72 (1960), 292-311.

Department of Mathematics, University of NoRth CaRolina at Charlotte, Charlotte, North CAROLINA 28223 